\title{
Cardiotoxicidad por quimioterapia en el Instituto Nacional de Enfermedades Neoplásicas 2012-2016
}

\author{
Enrique Ruiz-Mori ${ }^{1}$, Leonor Ayala-Bustamante ${ }^{1}$, Jorge Burgos-Bustamante ${ }^{1}$, Cristian Pacheco Román ${ }^{2}$
}

RESUMEN

Objetivo: Reportar la frecuencia de los efectos adversos a nivel cardiaco (cardiotoxicidad) producida por la quimioterapia en los pacientes del Instituto Nacional de Enfermedades Neoplásicas en el período 2012-2016.

Materiales y métodos: El estudio realizado fue de tipo no experimental, descriptivo y retrospectivo. La población estudiada fueron los pacientes atendidos en el Servicio de Cardiología por presentar durante la quimioterapia molestias cardiovasculares.

Resultados: Se realizaron 985 evaluaciones por sintomatología cardiaca de pacientes en tratamiento antineoplásico. Entre los efectos adversos a nivel del corazón de la terapia oncológica, las arritmias cardiacas fueron las más frecuentes $(41,2 \%)$, en segundo lugar estuvieron los episodios de angina de pecho con un $18,7 \%$ y la insuficiencia cardiaca tuvo una frecuencia del 4,9\%. La bradicardia sinusal ha sido la arritmia más usual $(55,9 \%)$, seguida por la taquicardia sinusal sintomática $(17,7 \%)$ y, en tercer lugar, la fibrilación auricular $(12,0 \%)$.

Conclusiones: La arritmia cardiaca fue el efecto adverso más frecuente del tratamiento oncológico, siendo la bradicardia sinusal asintomática la de mayor prevalencia.

Palabras Clave: Cardiotoxicidad; quimioterapia; arritmia cardiaca; cardiooncología (Fuente: DESC BIREME).

\section{Chemotherapy-induced cardiotoxicity at the Instituto Nacional de Enfermedades Neoplásicas 2012-2016}

\section{ABSTRACT}

Objective: To report the frequency of cardiac adverse effects (cardiotoxicity) induced by chemotherapy in patients of the Instituto Nacional de Enfermedades Neoplásicas during the period 2012-2016.

Materials and methods: The study had a non-experimental, descriptive and retrospective design. The study population consisted of patients treated at the Cardiology Service due to cardiovascular discomfort during chemotherapy.

Results: Nine hundred eighty-five (985) evaluations were performed because of cardiac symptoms in patients undergoing antineoplastic treatment. Among the oncological treatment-related cardiovascular adverse effects, cardiac arrhythmias were the most frequent ones (41.2\%), episodes of angina pectoris were in the second place with $18.7 \%$, and heart failure had a frequency of $4.9 \%$. The most frequent arrhythmia was sinus bradycardia $(55.9 \%)$, followed by symptomatic sinus tachycardia (17.7\%) and, in the third place, atrial fibrillation (12.0\%).

Conclusions: Cardiac arrhythmia was the most frequent adverse effect induced by oncological treatment, with asymptomatic sinus bradycardia being the most prevalent one.

Keywords: Cardiotoxicity; chemotherapy; cardiac arrhythmia; cardio-oncology (Source: MeSH NLM).

1. Servicio de Cardiología, Instituto Nacional de Enfermedades Neoplásicas. Lima, Perú.

2. Departamento de Oncología, Instituto Nacional de Enfermedades Neoplásicas. Lima, Perú. 


\section{INTRODUCCIÓN}

En los últimos años el cáncer se está convirtiendo en una enfermedad crónica, producto de los progresos en prevención, detección temprana y tratamiento con nuevos fármacos y tecnología de avanzada ${ }^{(1)}$.

En el 2012 se diagnosticaron 14.1 millones de casos nuevos de cáncer a nivel mundial ocasionando 8,2 millones de fallecidos pero logrando sobrevivir 32,6 millones de sujetos al cabo de 5 años ${ }^{(2)}$; en América Latina la incidencia por cáncer es menor (tasa estandarizada por edad es de 163 por 100 mil) en comparación con Europa (264 por 100 mil) o con los EE UU (300 por 100 mil), pero produciendo una alta mortalidad ${ }^{(3)}$; siendo considerada en esta parte del continente como la tercera causa de muerte. En el Perú se registran 56 mil nuevos casos de cáncer al año y más de 25 mil fallecidos, convirtiéndola en la segunda causa de muerte en la población adulta, luego de las enfermedades cardiovasculares ${ }^{(4)}$.

Los nuevos antineoplásicos si bien están demostrando cada vez una mayor eficacia, sin embargo se está incrementando la frecuencia de sus efectos adversos a nivel cardiaco o cardiotoxicidad. El término cardiotoxicidad engloba diversas manifestaciones patológicas a nivel cardiovascular producto del tratamiento oncológico, siendo la más frecuente la insuficiencia cardiaca, que en algunos casos puede ser de grado avanzado y con alta mortalidad, como sucede por el uso de antraciclínicos ${ }^{(5,6)}$. Los reportes sobre insuficiencia cardiaca secundaria a quimioterapia registran una mortalidad 3,5 veces mayor, comparado a la producida por cardiomiopatías idiopáticas ${ }^{(7)}$.

La Sociedad Americana del Cáncer estima que para el 2024 la tasa de supervivencia en pacientes oncológicos será del $66 \%$, aproximadamente 19 millones de sobrevivientes ${ }^{(8)}$; también advierte que algunas de las nuevas terapias contra el cáncer que incrementa la supervivencia, ocasionen mortalidad pero por afecciones cardiovasculares. En el estudio prospectivo de corte transversal de Wise con 1853 adultos sobrevivientes de cáncer en la infancia, tenían un aumento de la prevalencia de afecciones cardiacas en 3-24\% en los sobrevivientes de 30-39 años y $10-37 \%$ en los mayores de 40 años ${ }^{(9)}$.
Ante esta realidad se han elaborado nuevas guías de estrategias en el manejo y control de cáncer, estableciendo un trabajo coordinado entre el oncólogo, el cardiólogo, el hematólogo, entre otros; con la finalidad de propiciar una mayor eficacia de los agentes antitumorales sin incrementar el riesgo de mortalidad cardiovascular.

El objetivo general del estudio es reportar la frecuencia de los efectos adversos, en los pacientes del Instituto Nacional de Enfermedades Neoplásicas (INEN) que recibieron tratamiento médico oncológico, en el período 2012-2016.

\section{MATERIALES Y MÉTODOS}

El estudio realizado fue de tipo no experimental, descriptivo y retrospectivo. La población estudiada fueron los pacientes atendidos en el Servicio de Cardiología, durante el quinquenio 2012-2016, del INEN.

Criterio de Inclusión: paciente oncológico que ha recibido por lo menos una dosis de quimioterapia oral o parenteral, referidos por presentar alguna molestia cardiovascular.

No se incluyeron a aquellos sujetos que recibían en forma concomitante radioterapia a nivel torácico, o que sean portadores de cardiopatía previa.

\section{RESULTADOS}

Durante el quinquenio 2012-2016, en el Servicio de Cardiología del INEN, se realizaron 62,767 evaluaciones cardiológicas, de las cuales 985 fueron referidas por algún síntoma cardiovascular luego de la administración de un fármaco antineoplásico; siendo más frecuente en el género femenino $(61,12 \%)$ (Figura 1$)$.

Sobre los efectos adversos de la terapia farmacológica antineoplásica a nivel del aparato cardiovascular, las arritmias cardiacas fueron las de mayor frecuencia $(41,2 \%)$, y en segundo lugar los episodios de angina de pecho (Angor Pectoris) con un 18,7\%. La insuficiencia cardiaca sintomática (Clase Funcional II a III) estuvo presente en un $4,9 \%$. Los eventos adversos que se han diagnosticado con mayor frecuencia en las mujeres son las arritmias

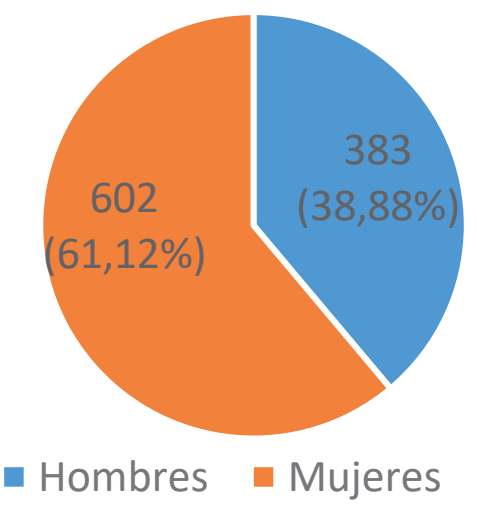

Figura 1. Número de pacientes atendidos por molestias cardiovasculares, según género 
cardiacas, el derrame pericárdico y la insuficiencia cardiaca, mientras que en los varones se presentaron con mayor frecuencia la angina de pecho, la hipertensión arterial y la trombosis venosa (Tabla 1).

Tabla 1. Efectos adversos sobre el corazón por quimioterapia

\begin{tabular}{|c|c|c|c|c|c|c|c|c|}
\hline & 2012 & 2013 & 2014 & 2015 & 2016 & & TOTAL & \\
\hline & & & & & & Hombres & Mujeres & Total \\
\hline $\begin{array}{l}\text { Arritmias } \\
\text { Cardiacas }\end{array}$ & 72 & 81 & 77 & 90 & 86 & 119 & 287 & $\begin{array}{c}406 \\
(41,2 \%)\end{array}$ \\
\hline $\begin{array}{l}\text { Angina de } \\
\text { Pecho }\end{array}$ & 31 & 35 & 39 & 45 & 34 & 102 & 82 & $\begin{array}{c}184 \\
(18,7 \%)\end{array}$ \\
\hline $\begin{array}{l}\text { Derrame } \\
\text { pericárdico }\end{array}$ & 15 & 23 & 30 & 32 & 43 & 60 & 83 & $\begin{array}{c}143 \\
(14,5 \%)\end{array}$ \\
\hline $\begin{array}{l}\text { Hipertensión } \\
\text { Arterial }\end{array}$ & 15 & 11 & 12 & 21 & 16 & 43 & 32 & $\begin{array}{c}75 \\
(7,6 \%)\end{array}$ \\
\hline $\begin{array}{l}\text { Insuficiencia } \\
\text { Cardiaca }\end{array}$ & 9 & 6 & 8 & 14 & 11 & 10 & 38 & $\begin{array}{c}48 \\
(4,9 \%)\end{array}$ \\
\hline $\begin{array}{l}\text { Trombosis } \\
\text { Venosa }\end{array}$ & 0 & 3 & 6 & 10 & 7 & 14 & 12 & $\begin{array}{c}26 \\
(2,6 \%)\end{array}$ \\
\hline Otras & 21 & 18 & 26 & 15 & 23 & 35 & 68 & $\begin{array}{c}103 \\
(10,5 \%)\end{array}$ \\
\hline TOTAL & 163 & 177 & 198 & 227 & 220 & $\begin{array}{c}383 \\
(38,88 \%)\end{array}$ & $\begin{array}{c}602 \\
(61,12 \%)\end{array}$ & $985(100 \%)$ \\
\hline
\end{tabular}

Entre las arritmias cardiacas evaluadas, la bradicardia sinusal ha sido la más frecuente (en un $55,9 \%$ ), la cual era asintomática en la mayoría de casos; en segundo lugar se detectó taquicardia sinusal sintomática $(17,7 \%)$ y en tercer lugar fibrilación auricular (12,0\%). Entre las arritmias cardiacas predominaron en la mujer la bradicardia sinusal, taquicardia sinusal y extrasistolia ventricular; mientras que la fibrilación auricular y la taquicardia paroxística supraventricular fueron más frecuente en los varones (Tabla 2).

Tabla 2. Arritmias cardiacas por quimioterapia

\begin{tabular}{|c|c|c|c|c|c|c|c|c|}
\hline & 2012 & 2013 & 2014 & 2015 & 2016 & & TOTAL & \\
\hline & & & & & & Hombres & Mujeres & Total \\
\hline $\begin{array}{l}\text { Bradicardia } \\
\text { sinusal }\end{array}$ & 30 & 41 & 50 & 58 & 48 & 45 & 182 & $\begin{array}{c}227 \\
(55,9 \%)\end{array}$ \\
\hline $\begin{array}{l}\text { Taquicardia } \\
\text { sinusal }\end{array}$ & 16 & 18 & 10 & 15 & 13 & 26 & 46 & $\begin{array}{c}72 \\
(17,7 \%)\end{array}$ \\
\hline $\begin{array}{l}\text { Fibrilación } \\
\text { auricular }\end{array}$ & 7 & 9 & 8 & 11 & 14 & 27 & 22 & $\begin{array}{c}49 \\
(12,0 \%)\end{array}$ \\
\hline $\begin{array}{l}\text { Extrasistolia } \\
\text { Ventricular }\end{array}$ & 10 & 6 & 5 & 3 & 8 & 11 & 21 & $\begin{array}{c}32 \\
(7,9 \%)\end{array}$ \\
\hline $\begin{array}{l}\text { Taquicardia } \\
\text { Paroxística } \\
\text { Supraventricular }\end{array}$ & 4 & 2 & 1 & 1 & 0 & 5 & 3 & $\begin{array}{c}8 \\
(2,0 \%)\end{array}$ \\
\hline Otras & 5 & 5 & 3 & 2 & 3 & 5 & 13 & $\begin{array}{c}18 \\
(4,4 \%)\end{array}$ \\
\hline TOTAL & 72 & 81 & 77 & 90 & 86 & 119 & 287 & $\begin{array}{c}406 \\
(100 \%)\end{array}$ \\
\hline
\end{tabular}




\section{DISCUSIÓN}

La presencia del mayor número de mujeres refiriendo eventos adversos en el estudio, probablemente se explique considerando que las mujeres acuden con mayor frecuencia $(62,9 \%)$ por atención de salud al INEN que los varones ${ }^{(10)}$.

Los efectos adversos a nivel cardiovascular por quimioterapia en los últimos años han tenido un marcado incremento en frecuencia, sobre todo con la aparición de nuevos fármacos antineoplásicos. El clásico daño del miocardio por la quimioterapia se conoce desde hace medio siglo, cuando en 1967 se reportaron los primeros casos de insuficiencia cardiaca en niños con leucemia que recibían altas dosis de antraciclínicos ${ }^{(11)}$.

La principal causa por la que se solicitó evaluación cardiológica en los pacientes oncológicos del INEN entre el 2012 al 2016, fue la arritmia cardiaca, que incluye una amplia gama de patologías, desde las bradi-arritmias hasta las taqui-arritmias, englobando a la fibrilación auricular, taquicardias paroxísticas, entre otras. Los antraciclínicos y taxanos, son los medicamentos que más han propiciado los trastornos del ritmo cardiaco ${ }^{(12)}$. Hay una serie de condiciones, aparte del medicamento oncológico (dosis, forma de administración, velocidad de infusión, etc.), que determinan la presencia de arritmias como son la edad, historia de hipertensión arterial, de cardiopatía subyacente, etc. En la presente serie, la principal arritmia que determinó la evaluación cardiológica fue la bradicardia sinusal, la cual generalmente fue asintomática, pero que obligó en algunos casos a realizar medidas de seguridad a fin de continuar el tratamiento o el uso de drogas beta-agonistas. En ningún caso fue necesario el uso de marcapaso. Los antraciclínicos desarrollan en forma transitoria durante o inmediatamente después de la administración arritmias, considerándose como toxicidad aguda; los taxanos, fármacos que actúan directamente sobre los microtubulis, clásicamente están asociados a bradicardia; sin embargo recientemente conforme se utiliza cada vez más trastuzumab, se está empezando a encontrar con frecuencia bradicardia sinusal asintomática. La taquicardia sinusal que motivó la evaluación por cardiología ha sido sólo la sintomática (disnea, fatiga, palpitaciones) y que requirió tratamiento médico. La tercera arritmia en frecuencia fue la fibrilación auricular, siendo más frecuente en varones (probablemente relacionado a que en el presente estudio la hipertensión arterial ha sido más frecuente en los hombres). Con relativa frecuencia se observan cambios en el segmento ST y alteraciones dinámicas de la onda T en forma difusa.

Los fenómenos de vasoespasmo, que con relativa periodicidad se observa con el uso de 5-Fluorouracilo y que condiciona Angina de Pecho ${ }^{(13)}$; en los últimos años, productos de los nuevos, pero desfavorables, estilos de vida; la enfermedad coronaria isquémica se presenta a edad más temprana y se ha convertido en la principal causa de muerte de la población adulta, incluso en el Perú. La mayor prevalencia de ateroesclerosis facilita que un paciente oncológico que está indicado administrarle 5-Fluorouracilo, el fenómeno vasoespástico se produzca en una arteria con un grado de ateroesclerosis, agravando la isquemia y propiciando un mayor riesgo coronario. En el presente estudio el angor pectoris es la segunda causa que motiva la evaluación por cardiología en relación a los efectos adversos de la quimioterapia.

La presencia de pericarditis en un paciente con cáncer, inicialmente obliga a considerar como extensión de la neoplasia y ser un signo de alarma; cada vez se están reportando casos de efusión pericárdica como consecuencia de la quimioterapia. Los agentes oncológicos como los antimetabolitos (Citarabina), los antraciclínicos (Daunorubicina) y los agentes alquilantes (Ciclofosfamida), entre sus eventos adversos se reportan casos de pericarditis, que pueden acompañarse de derrame pericárdico de variada magnitud, que en ciertas ocasiones puede desencadenar taponamiento cardiaco o propiciar pericarditis crónica constrictiva. En el quinquenio en estudio, el derrame pericárdico fue el tercer efecto colateral del tratamiento oncológico sobre el corazón, no habiéndose desarrollado ningún caso de pericarditis constrictiva ${ }^{(14)}$.

La adriamicina, corresponde a un grupo de fármacos (Antraciclínicos y Antraquinonas) de reconocida eficacia, con indicación en múltiples neoplasias sólidas (cáncer de mama, sarcomas de partes blandas y hueso, entre otros) y hematopoyéticas (leucemias, linfomas, etc.); sin embargo el mayor limitante en su uso y efectividad es la toxicidad que se puede producir en el músculo cardiaco producto de la alta generación de radicales libres y la presencia de Topoisomerasas $2 B$, produciendo una lisis o apoptosis de los cardiomiocitos, en forma global o difusa, condicionando una Cardiomiopatía Dilatada, que conlleva a la insuficiencia cardiaca sistólica (IC), la cual es irreversible y con alta mortalidad ${ }^{(15,16)}$. En el estudio actual se han detectado en el lapso de los últimos cinco años 48 nuevos casos, que presentaban sintomatología y signología de Insuficiencia Cardiaca (Clase Funcional II y III de la NYHA), dos de ellos habiendo presentado Edema Agudo Pulmonar y con evolución tórpida. Entre las medidas para evitar la cardiotoxicidad por antraciclínicos es el cuidado en no alcanzar dosis elevadas que pongan en riesgo la vida del paciente, teniendo en cuenta que otras medidas como las de usar nuevas preparaciones liposomales son de muy alto costo y por lo tanto de difícil acceso a la población (17). En el trabajo la frecuencia de insuficiencia cardiaca fue cuatro veces mayor en las mujeres que en los hombres, esto se explica por el uso oncológico de los antraciclínicos que se realiza en el INEN sobre todo en cáncer de mama.

En los últimos años un nuevo grupo de drogas antitumorales denominados antimonoclonales como el trastuzumab, se encuentra utilizándose sobre todo en cáncer de mama metastásico HER2 positivo, con mayores y claros beneficios, pero también conlleva a un compromiso de la función miocárdica, pero de forma reversible, con pronóstico y manejo muy diferente a la tradicional cardiotoxicidad de los antraciclínicos. El mecanismo de este fármaco sobre el corazón es un bloqueo de los receptores HER2 de la superficie de los cardiomiocitos, lo que causa una depleción de ATP, ocasionando una disfunción contráctil del corazón; este efecto cardiodepresor no es dosis dependiente y es reversible, se revierte en dos a tres semanas. Generalmente se produce una reducción de la función ventricular izquierda asintomática, la cual rara vez evoluciona hacia una franca insuficiencia cardiaca ${ }^{(18,19)}$.

Si bien la tecnología médica avanza (strain bidimensional, ecocardiografía tridimensional con contraste) y permite evaluaciones muy minuciosas, aún la ecocardiografía sigue siendo un método no invasivo, de bajo costo y de gran utilidad en el control, manejo y seguimiento de los pacientes con cáncer que reciben fármacos y/o radioterapia que pueden afectar al miocardio. La determinación de la fracción de eyección del ventrículo izquierdo por la ecocardiografía 
antes, durante y después de la quimioterapia es el método más accesible y económico. También puede brindar la ecocardiografía una serie de informaciones sobre la función diastólica del ventrículo izquierdo, el tamaño de las cámaras ventriculares, el grosor de las paredes, así como el estado del aparato valvular y subvalvular ${ }^{(20,21)}$. De tal forma que esta herramienta seguirá teniendo vigencia en los protocolos de diagnóstico y seguimiento de la cardiotoxicidad.

Es una necesidad actual contar con guías de consenso en el diagnóstico, prevención y tratamiento de la cardiotoxicidad por oncoterapia médica, permitiendo que los tratamientos con quimioterapia fuesen más seguros y alcancen cifras importantes de eficacia, ampliando la sobrevida del paciente oncológico. Fomentar la constitución de equipos o unidades de Cardio-Oncología para el manejo del cáncer en forma multidisciplinaria, integrando al oncólogo clínico, pediatra-oncólogo, radioterapista, hematólogo, cardiólogo y especialistas afines; a fin de establecer el riesgo cardiovascular, plantear de acuerdo a ello la terapia más adecuada y realizar una vigilancia estrecha del tratamiento del cáncer sobre el aparato cardiovascular; deberá ser una exigencia.

En conclusión, en el presente trabajo la arritmia cardiaca es el efecto adverso más frecuente del tratamiento oncológico, siendo la bradicardia sinusal asintomática la de mayor prevalencia.

Contribuciones de los autores: ER, LA, JB y CP participaron en la concepción, realización, redacción y revisión de la versión final.

\section{REFERENCIAS BIBLIOGRÁFICAS}

1. Brenner H, Francisci S, De Angelis R, Marcos-Gragera R, Verdecchia A, Gatta G, et al. Long term survival expectations of cancer patients in Europe in 2000-2002. Eur J Cancer. 2009; 45(6):1028-41.

2. World Health Organization. Globocan 2012: Estimated Cancer incidence, mortality and prevalence worldwide. Lyon: International Agency for Research on Cancer; 2012.

3. Ministerio de Salud. Análisis de la situación del cáncer en el Perú 2013. Lima: Dirección general de epidemiologia; 2013.

4. Salazar M, Regalado-Rafael R, NavarroJ, Montanez D, Abugattas J, Vidaurre T. El Instituto Nacional de Enfermedades Neoplásicas en el Control del Cáncer en el Perú. Rev Peru Med Exp Salud Pública. 2013;30(1):105-12.

5. Ruiz E, Ayala L, Burgos J. Insuficiencia Cardiaca por quimioterapia. Rev Soc Peruana Med Interna. 2016;29(2):59-64.

6. Perrino C, Schiattarella G, Magliulo F, Ilardi F, Carotenuto G, Gargiulo G, et al. Cardiac side effects of chemotherapy: state of art and strategies for a correct management. Curr Vasc Pharmacol. 2014;12(1):106-16.

7. Bloom M, Hamo C, Cardinale D, Ky B, Nohria A, Baer L, et al. Cancer Therapy-Related Cardiac Dysfunction and Heart Failure. Circ Heart Fail. 2016;9(2):e002843.

8. Okwuosa T, Yakupovich A. Cardio-Oncology Programs in the United States. International Journal of Collaborative Research on Internal Medicine \& Public Health. [Internet] 2016. [acceso Julio del 2017];8(4). Disponible en: http://internalmedicine.imedpub. com/cardiooncology-programs-in-the-united-states.pdf

9. Wise J. Adult survivors of childhood cancer are at high risk of cardiac abnormalities, study finds. BMJ. 2016;352: 7026.

10. Ministerio de Salud. Registro hospitalario de cáncer en el INEN en Lima - Perú 2000-2012.Lima: Instituto Nacional de Enfermedades Neoplasicas; 2016.

11. Navarrete S, Castellanos A, Chaparro A. Cardiotoxicidad por quimioterapia. Un enfoque práctico para el clínico. Insuficiencia Cardiaca. [Internet] 2011. [acceso Julio del 2017];6(3). Disponible en: http://www.scielo.org.ar/scielo. php?script=sci_arttext\&pid=S1852-38622011000300005

12. Lax J, Piñero $D$, Falconi $M$, Agüero $R$, Barugel $M$, Bermann $A$, et al. Consenso de diagnóstico, prevención y tratamiento de la cardiotoxicidad por tratamiento médico del cáncer. Rev argent cardiol [Internet]. 2013 [acceso Julio del 2017];81(6). Disponible en: http://www. scielo.org. ar/scielo.php?script=sci_arttext\&pid=S1850$37482013000600012 \& \operatorname{lng}=$ es.

13. López-Velarde T, Lopez-Velarde P. Prevención y tratamiento de cardiotoxicidad en pacientes con cáncer. An Med (Mex). 2014;59(1):54-60.

14. Bock J, Doenitz A, Andreesen R, Reichle A, Hennemann B. Pericarditis after high-dose chemotherapy: more frequent than expected?. Onkologie. 2006;29(7):321-4.

15. Cárdenas R. Mecanismos de cardiotoxicidad de la quimioterapia. GAMO. 2009; 8(3):3-7.

16. Parma G, Lluberas N, Castillo C, Ormaechea G. Quimioterápicos y Cardiotoxicidad: un enfoque actual y práctico para el clínico de una disciplina en pleno desarrollo. Arch Med Int [Internet].2013[citado Julio del 2017];35(2):38-47.Disponible en: $\quad$ http://www.scielo.edu.uy/scielo.php?script=sci_ arttext\&pid=S1688-423X2013000200003\&lng=es.

17. Velásquez C, González M, Berrouet M, Jaramillo N. Cardiotoxicidad inducida por la quimioterapia desde las bases moleculares hasta la perspectiva clínica. Rev Colomb Cardiol. 2016;23(2):104-11.

18. Serrano C, Cortés J, De Mattos-Arruda L, Bellet M, Gómez $P$, Saura C, et al. Trastuzumab-related cardiotoxicity in the elderly: a role for cardiovascular risk factors. Ann Oncol. 2012;23(4):897-902.

19. Chien K. Herceptin and the heart. A molecular modifier of cardiac failure. N Engl J Med. 2006;354(8):789-90.

20. Florenzano F, Venegas P. Prevención de la cardiotoxicidad en pacientes que reciben quimioterapia. Rev Med Clin Condes. 2012;23(6):772-81.

21. Plana J. La quimioterapia y el corazón. Rev Esp Cardiol. 2011;64(5):409-15.

\section{Fuentes de financiamiento:}

Este artículo ha sido financiado por los autores.

Conflictos de interés:

Los autores declaran no tener ningún conflicto de interés.

\section{Correspondencia:}

Enrique Ruiz Mori

Dirección: Av. Angamos 2520 Lima 34 Perú.

Teléfono: (511)998788580.

Correo electrónico: cruizm@usmp.pe

Recibido: 01 de agosto de 2017 Evaluado: 03 de agosto de 2017 Aprobado: 14 de agosto de 2017

(C) La revista. Publicado por Universidad de San Martín de Porres, Perú. (cc) в bajo términos de Licencia Creative Commons Atribución 4.0 Internacional. (http: //creativecommons.org/licenses/by/4.0/) 